\title{
Chiral Symmetry Restoration in Holographic Noncommutative QCD
}

\author{
Tadahito NAKAJIMA, ${ }^{*}$ Yukiko OHTAKE ${ }^{\dagger}$ and Kenji SUZUKI ${ }^{\ddagger}$
}

\author{
* College of Engineering, Nihon University, Fukushima 963-8642, Japan \\ $\dagger$ Toyama National College of Technology, Toyama 939-8630, Japan \\ $\ddagger$ Department of Physics, Ochanomizu University, Tokyo 112-8610, Japan
}

\begin{abstract}
We consider the noncommutative deformation of the Sakai-Sugimoto model at finite temperature and finite baryon chemical potential. The space noncommutativity is possible to have an influence on the flavor dynamics of the QCD. The critical temperature and critical value of the chemical potential are modified by the space noncommutativity. The influence of the space noncommutativity on the flavor dynamics of the QCD is caused by the Wess-Zumino term in the effective action of the D8-branes. The intermediate temperature phase, in which the gluons deconfine but the chiral symmetry remains broken, is easy to be realized in some region of the noncommutativity parameter.
\end{abstract}




\section{Introduction}

Noncommutative gauge theories (gauge theories on noncommutative space) naturally arise as low energy theories of D-branes in Neveu-Schwarz-Neveu-Schwarz (NS-NS) B-field background [1, 2, 3, 4, 5]. The space noncommutativity brings nontrivial properties on the gauge field theory at the quantum level. A remarkable example is the mixing between the infrared and the ultraviolet degrees of freedom. Although the product of the momentum and the noncommutativity parameter plays the role of ultraviolet cut-off, the result is singular when the momentum or the noncommutativity parameter is taken to zero. This phenomenon is referred to as the UV/IR mixing [6]. Although the noncommutative gauge theories have been studied extensively, it is hard to investigate them in the perturbative approach. Little is known of the non-parturbative information of noncommutative gauge theories.

The noncommutative gauge theories have gravity duals whose near horizon region describes the large $N$ limit of the noncommutative gauge theories. This is first constructed by Hashimoto and Itzhaki [7], and Maldacena and Russo [8]. Using the gravity dual description, we can investigate the non-perturbative aspects of the large $N$ limit of the noncommutative gauge theories. For instance, the space noncommutativity modifies the Wilson loop behavior [10, 11, 12 and glueball mass spectra [13]. The gravity duals of noncommutative gauge theories with matter in the fundamental representation have also been constructed by adding probe flavor branes [14]. Throughout the construction of gravity duals of noncommutative gauge theories with matter degrees of freedom it has been found the space noncommutativity is also reflected in the flavor dynamics. For instance, the mass spectrum of mesons can be modified by the space noncommutativity [14].

The gauge/gravity correspondence can be developed to study the physics of chiral symmetry breaking, besides confinement in low energy behaviors of quantum chromodynamics (QCD) [15, 16, 17]. An attempt to describe the low energy hadron physics in terms of the gauge/gravity correspondence is called the holographic QCD model. The gravity duals of theories with nonAbelian chiral symmetry have been constructed by Sakai and Sugimoto [18, 19]. This model, so

called Sakai-Sugimoto model, is known as one of the most accurate holographic realizations of the real QCD. The holographic QCD models can be modified to introduce a finite temperature and a finite baryon chemical potential. In the Sakai-Sugimoto model, phase transition of chiral symmetry restoration has been investigated at finite temperature in [20, 21, 22, 23] and finite baryon chemical potential in [24, 25, 26]. The phase diagrams of holographic QCD have been shown to contain a line of first order phase transitions.

The presence of magnetic field promotes the spontaneous chiral symmetry breaking in QCD. The effect of an external constant $B$-field in the Sakai-Sugimoto model on the phase structure of the QCD has been investigated and it has been shown that the external constant $B$-field promotes chiral symmetry breaking in the QCD [31, 32, 33]. This fact seems to suggest that 
NS-NS B-field background, that causes the space noncommutativity in the QCD, has a certain effect on the chiral phase transition of the QCD.

There have been some papers on how to influence the NS-NS $B$-field background on the strong coupled dynamics of the QCD in the holographic approach. The influence of the NSNS $B$-field background on the drag force in thermal plasma of Yang-Mills theories has been investigated in [28, 29, 30]. The influence of the NS-NS $B$-field background on the chiral symmetry restoration in the QCD at finite temperature has also been investigated in the manner of adding a constant background magnetic field besides the NS-NS B-field in Sakai-Sugimoto model [27].

In this paper, we construct the noncommutative deformation of the Sakai-Sugimoto model in imitation of the method by Arean et al. [14] and investigate the influence of the NS-NS $B$-field background on the chiral symmetry restoration in the QCD at finite temperature and finite baryon chemical potential. As will be seen later, the introduction of the Wess-Zumino (WZ) term produces the dependence of the effective action of the probe D8-brane on the NS-NS $B$-field background. The existence of the gauge fields in the total effective action of the probe D8-brane carries the dependence of the baryon chemical potential on the QCD.

This paper is organized as follows. In section 2, we construct the noncommutative deformation of the holographic QCD model at finite temperature and finite baryon chemical potential. In section 3, we investigate the chiral phase transition in the noncommutative QCD within the framework of the noncommutative deformation of the holographic QCD model. Section 4 is devoted to conclusions and discussions.

\section{Noncommutative deformation of the holographic QCD model at finite temperature and finite baryon chemical potential}

In this section, we consider a noncommutative deformation of the holographic QCD (SakaiSugimoto) model at finite temperature based on the prescription of Arean-Paredes-Ramallo [14]. The holographic QCD model is a gravity dual for a $4+1$ dimensional QCD with $\left.\mathrm{U}_{(} \mathrm{N}_{f}\right)_{L} \times$ $\mathrm{U}\left(\mathrm{N}_{f}\right)_{R}$ global chiral symmetry whose symmetry is spontaneously broken [18, 19]. This model is a D4-D8- $\overline{\mathrm{D} 8}$-brane system consisting $S^{1}$ compactified $N_{c}$ D4-branes and $N_{f}$ D8-D8 pairs transverse to the $S^{1}$. The near-horizon limit of the set of $N_{c}$ D4-branes solution compactified 
on $S^{1}$ takes the form

$$
\begin{aligned}
d s^{2} & =\left(\frac{U}{R_{\mathrm{D} 4}}\right)^{3 / 2}\left(-(d t)^{2}+\left(d x^{1}\right)^{2}+\left(d x^{2}\right)^{2}+\left(d x^{3}\right)^{2}+f(U) d \tau^{2}\right) \\
& +\left(\frac{R_{\mathrm{D} 4}}{U}\right)^{3 / 2}\left(\frac{d U^{2}}{f(U)}+U^{2} d \Omega_{4}^{2}\right) \\
& R_{\mathrm{D} 4}^{3}=\pi g_{s} N_{c} l_{s}^{3}, \quad f(U)=1-\frac{U_{\mathrm{KK}}{ }^{3}}{U^{3}},
\end{aligned}
$$

where $U_{\mathrm{KK}}$ is a parameter, $U$ is the radial direction bounded from below by $U \geq U_{\mathrm{KK}}, \tau$ is compactified direction of the D4-brane world volume which is transverse to the D8-D8-branes, $g_{s}$ and $l_{s}$ are the string coupling and length respectively. The dilaton $\phi$ and the field strength $F_{4}$ of the RR 3-form $C_{3}$ are given by

$$
e^{\phi}=g_{s}\left(\frac{U}{R_{\mathrm{D} 4}}\right)^{3 / 4}, \quad F_{4}=d C_{3}=\frac{2 \pi N_{c}}{V_{4}} \epsilon_{4},
$$

where $V_{4}=8 \pi^{2} / 3$ is the volume of unit $S^{4}$ and $\epsilon_{4}$ is the corresponding volume form. In order to avoid a conical singularity at $U=U_{\mathrm{KK}}$, the $\tau$ direction should have a period of

$$
\delta \tau=\frac{4 \pi}{3}\left(\frac{R_{\mathrm{D} 4}^{3}}{U_{\mathrm{KK}}}\right)^{1 / 2}=2 \pi R=\frac{2 \pi}{M_{\mathrm{KK}}},
$$

where $R$ is radius of $S^{1}$ and $M_{\mathrm{KK}}$ is the Kaluza-Klein mass. The parameter $U_{\mathrm{KK}}$ is related to the Kaluza-Klein mass $M_{\mathrm{KK}}$ via the relation (2.3). The five dimensional gauge coupling is expressed in terms of $g_{s}$ and $l_{s}$ as $g_{\mathrm{YM}}^{2}=(2 \pi)^{2} g_{s} l_{s}$. The gravity description is valid for strong coupling $\lambda \gg R$, where as usual $\lambda=g_{\mathrm{YM}}^{2} N_{c}$ denotes the 't Hooft coupling.

The holographic QCD model at finite temperature has been proposed in [20, 21, 22]. In order to introduce a finite temperature $T$ in the model, we consider the Euclidean gravitational solution which is asymptotically equals to (2.1) but with the compactification of Euclidean time direction $t_{E}$. In this solution the periodicity of $t_{E}$ is arbitrary and equals to $\beta=1 / T$.

Another solution with the same asymptotic is given by interchanging the role of $t_{E}$ and $\tau$ directions,

$$
\begin{aligned}
d s^{2} & =\left(\frac{U}{R_{\mathrm{D} 4}}\right)^{3 / 2}\left(\tilde{f}(U)\left(d t_{E}\right)^{2}+\left(d x^{1}\right)^{2}+\left(d x^{2}\right)^{2}+\left(d x^{3}\right)^{2}+d \tau^{2}\right) \\
& +\left(\frac{R_{\mathrm{D} 4}}{U}\right)^{3 / 2}\left(\frac{d U^{2}}{\widetilde{f}(U)}+U^{2} d \Omega_{4}^{2}\right), \\
& R_{\mathrm{D} 4}^{3}=\pi g_{s} N_{c} l_{s}^{3}, \quad \tilde{f}(U)=1-\frac{U_{T}^{3}}{U^{3}},
\end{aligned}
$$

where $U_{T}$ is a parameter. To avoid a singularity at $U=U_{T}$ the period of $\delta t_{E}$ of the compactified time direction is set to

$$
\delta t_{E}=\frac{4 \pi}{3}\left(\frac{R_{\mathrm{D} 4}^{3}}{U_{T}}\right)^{1 / 2}=\frac{1}{T},
$$


and the parameter $U_{T}$ is related to the temperature $T$. The metric (2.1) with the compactification of Euclidean time $t_{E}$ is dominant in the low temperature $T<1 / 2 \pi R$, while the metric (2.4) is dominant in the high temperature $T>1 / 2 \pi R$. The transition between the metric (2.1) and the metric (2.4) happens when $T=1 / 2 \pi R$. This transition is first-order and corresponds to the confinement/deconfinement phase transition in the gauge theory side.

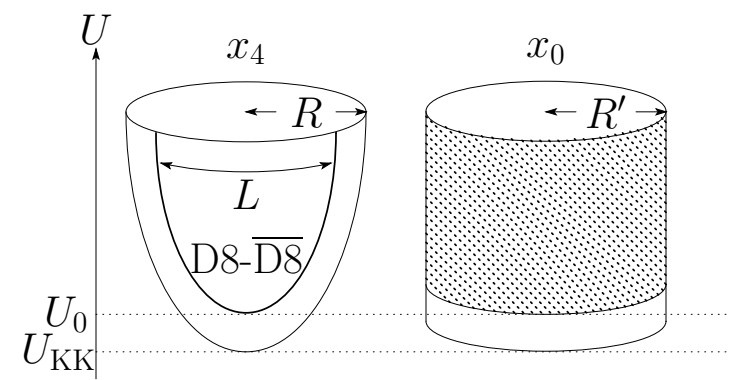

Fig. 1 The D8-D8-branes configurations at low temperature.
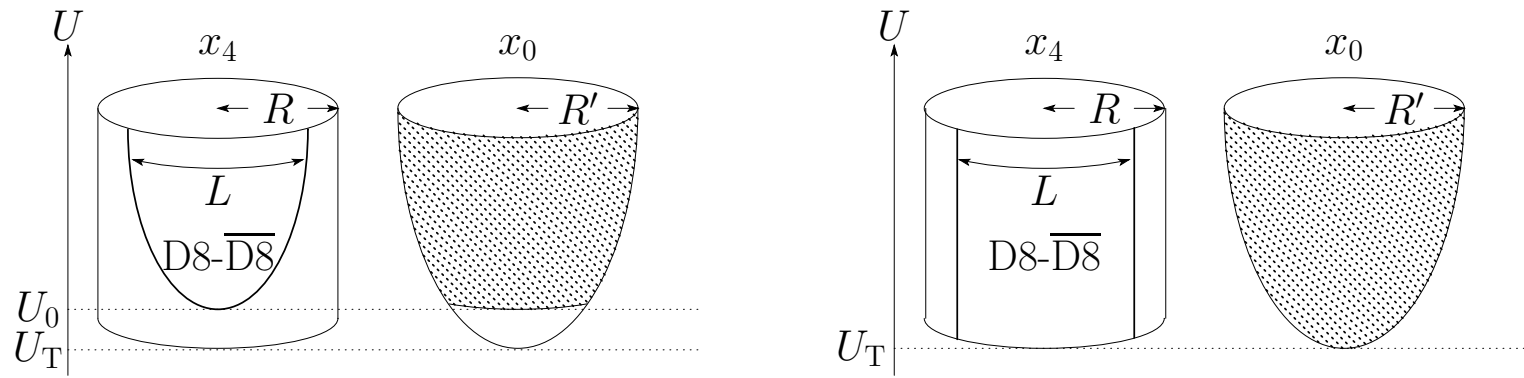

Fig. 2 The D8- $\overline{\mathrm{D} 8}$-branes configurations at high temperature.

In the low temperature, the D8- and $\overline{\mathrm{D} 8}$-branes are connected at $U=U_{0}$ as shown in Fig. 1. The connected configuration of the $\mathrm{D} 8-\overline{\mathrm{D}} 8$-branes indicates that the $\mathrm{U}\left(\mathrm{N}_{f}\right)_{L} \times \mathrm{U}\left(\mathrm{N}_{f}\right)_{R}$ global chiral symmetry is broken to a diagonal subgroup $\mathrm{U}\left(\mathrm{N}_{f}\right)$. We refer to the connected configuration in the low temperature as the low-temperature phase. In the high temperature, there are two kinds of configurations as shown in Fig. 2. One is connected configuration and the other is disconnected configuration that the D8- and $\overline{\mathrm{D} 8}$-branes hang vertically from infinity down to the horizon. The disconnected configuration of the D8- $\overline{\mathrm{D}} 8$-branes indicates that the $\mathrm{U}\left(\mathrm{N}_{f}\right)_{L} \times \mathrm{U}\left(\mathrm{N}_{f}\right)_{R}$ global chiral symmetry is restored. We refer to the disconnected configuration and the connected configuration in the high temperature as the high-temperature phase and the intermediate-temperature phase, respectively. The intermediate-temperature phase is realized when the confinement/deconfinement phase transition and the chiral phase transition does not occur simultaneously. If the ratio $L / R$ is larger than 0.97 , there is no intermediate-temperature phase [20].

When turning on a NS-NS $B$-field on the D-brane worldvolume, the low-energy effective 
worldvolume theories are deformed to a noncommutative Yang-Mills theories [1, 2, 3, 4, 5]. The D-brane realizations of noncommutative Yang-Mills theories have a gravity dual in the large $N$, strong 't Hooft coupling limit [7, 8, 9]. In accordance with the formulation of [7, 8, 9], we attempt to construct the gravity dual of the noncommutative QCD whose chiral symmetry is spontaneously broken by deforming the holographic QCD model. Let us consider the D4branes solution compactified on a circle in the $\tau$-direction. T-dualizing it along $x^{3}$ produces a D3-branes delocalized along $x^{3}$. After rotating the D3-branes along the $\left(x^{2}, x^{3}\right)$ plane, we T-dualize back on $x^{3}$. This procedure yields the solution with a $B_{23}$ fields along the $x^{2}$ and $x^{3}$ directions. The solution in the low temperature takes the form

$$
\begin{aligned}
d s^{2} & =\left(\frac{U}{R_{\mathrm{D} 4}}\right)^{3 / 2}\left(\left(d t_{E}\right)^{2}+\left(d x^{1}\right)^{2}+h\left\{\left(d x^{2}\right)^{2}+\left(d x^{3}\right)^{2}\right\}+f(U) d \tau^{2}\right) \\
& +\left(\frac{R_{\mathrm{D} 4}}{U}\right)^{3 / 2}\left(\frac{d U^{2}}{f(U)}+U^{2} d \Omega_{4}^{2}\right)
\end{aligned}
$$

where $h(U)=\frac{1}{1+\theta^{3} U^{3}}$ and $\theta$ denotes the noncommutativity parameter with dimension of mass. When $\theta \neq 0$ this solution is dual to a gauge theory in which the coordinates $x^{2}$ and $x^{3}$ do not commute. It is obvious that this solution reduces to the solution (2.1) with Euclidean signature when $\theta=0$. In the high temperature, the solution (2.6) changes to

$$
\begin{aligned}
d s^{2} & =\left(\frac{U}{R_{\mathrm{D} 4}}\right)^{3 / 2}\left(\tilde{f}(U)\left(d x_{E}\right)^{2}+\left(d x^{1}\right)^{2}+h\left\{\left(d x^{2}\right)^{2}+\left(d x^{3}\right)^{2}\right\}+d \tau^{2}\right) \\
& +\left(\frac{R_{\mathrm{D} 4}}{U}\right)^{3 / 2}\left(\frac{d U^{2}}{\tilde{f}(U)}+U^{2} d \Omega_{4}^{2}\right) .
\end{aligned}
$$

The solution has the same form as the one in the low temperature (2.6), but with the role of the $\tau$ and $t_{E}$ directions exchanged.

The effective action of the D8 branes is given as the Dirac-Born-Infeld(DBI) action with Wess-Zumino-like(WZ) term,

$$
\begin{aligned}
& S_{\mathrm{D} 8}=S_{\mathrm{D} 8}^{\mathrm{DBI}}+S_{\mathrm{D} 8}^{\mathrm{WZ}} \\
& S_{\mathrm{D} 8}^{\mathrm{DBI}}=T_{8} \int d^{9} x e^{-\phi} \operatorname{Tr} \sqrt{\operatorname{det}\left(g_{M N}+B_{M N}+2 \pi l_{s}^{2} F_{M N}\right)}, \\
& S_{\mathrm{D} 8}^{\mathrm{WZ}}=T_{8} \int_{\mathrm{D} 8} C_{3} \wedge e^{\left(B+2 \pi l_{s}^{2} F\right)},
\end{aligned}
$$

where $T_{8}=1 /(2 \pi)^{8} l_{s}^{9}$ is the tension of the D8 brane, $g_{M N}$ and $F_{M N}(M, N=0,1, \ldots, 8)$ are the induced metric and the field strength of the $U\left(N_{f}\right)$ gauge field $A_{M}$ on the D8-brane, respectively. We regard the gauge field on the D8-brane as a background gauge field. For simplicity, we assume that $A_{0}$ and $A_{1}$ components of the $U(1)$ part of the gauge field are 
non-vanishing fields. As will be mentioned later, the boundary value of $A_{0}$ is related to the baryon number chemical potential. The $B$-field is not a constant field which promotes chiral symmetry breaking in the QCD [31, 32, 33]. The notation $B_{M N}$ denotes the pullback of the NS-NS B-field :

$$
B_{M N}(U)=\left\{\begin{array}{ll}
\theta^{3 / 2} \frac{U^{3}}{R_{\mathrm{D} 4}^{3 / 2}} h(U) & (M=2, N=3) \\
0 & \text { (others) }
\end{array} .\right.
$$

We make an ansatz that $A_{0}$ and $\tau$ depend only on the coordinate $U$ :

$$
\tau=\tau(U), \quad A_{0}=A_{0}(U)
$$

and $A_{1}$ is not an auxiliary field but a non-zero constant.

\section{Chiral phase restoration in the noncommutative QCD}

\subsection{Low temperature}

In order to determine the chiral phase of the noncommutative QCD, we need to analyze the shape of the probe D8-branes. The induced metric on the probe D8-branes associated with the confining phase reads

$$
\begin{aligned}
d s^{2} & =\left(\frac{U}{R_{\mathrm{D} 4}}\right)^{3 / 2}\left(\left(d t_{E}\right)^{2}+\left(d x^{1}\right)^{2}+h\left\{\left(d x^{2}\right)^{2}+\left(d x^{3}\right)^{2}\right\}\right)+\left(\frac{R_{\mathrm{D} 4}}{U}\right)^{3 / 2} U^{2} d \Omega_{4}^{2} \\
& +\left[\left(\frac{U}{R_{\mathrm{D} 4}}\right)^{3 / 2} f(U)\left(\tau^{\prime}(U)\right)^{2}+\left(\frac{R_{\mathrm{D} 4}}{U}\right)^{3 / 2} \frac{1}{f(U)}\right] d U^{2},
\end{aligned}
$$

where $\tau^{\prime}=\frac{d \tau}{d U}$. Substituting the determinant of the induced metric (3.1) and the dilaton in the $B$-field background $e^{\phi}=g_{s} h^{1 / 2}\left(\frac{U}{R_{\mathrm{D} 4}}\right)^{3 / 4}$ into the DBI action (2.8), we have

$$
S_{\mathrm{D} 8}^{\mathrm{DBI}}=\frac{N_{f} T_{8} V_{4}}{g_{s}} \int d^{4} x d U U^{4} \sqrt{f \tau^{\prime 2}+\left(\frac{R_{\mathrm{D} 4}}{U}\right)^{3}\left(f^{-1}-\left(2 \pi l_{s}^{2} A_{0}^{\prime}\right)^{2}\right)},
$$

where $A_{0}^{\prime}=\frac{d A_{0}}{d U}$. We notice that the $B$-field in the DBI determinant $\operatorname{det}\left(g_{M N}+B_{M N}+\right.$ $\left.2 \pi l_{s}{ }^{2} F_{M N}\right)$ is dropped out and there is no dependence on the noncommutativity parameter $\theta$ in the DBI action. The independence of the DBI action on the noncommutativity parameter also takes place in the effective action of the probe D7-brane [14]. Adding the relevant WZ-term

$$
S_{\mathrm{D} 8}^{\mathrm{WZ}}=T_{8} \frac{\left(2 \pi l_{s}\right)^{2}}{2} \int_{\mathrm{D} 8} C_{3} \wedge B \wedge F \wedge F
$$


to the DBI action and using the relation (2.2), we find the term involving the $B$-field in the effective action of the D8-branes

$$
\begin{aligned}
S_{\mathrm{D} 8} & =S_{\mathrm{D} 8}^{\mathrm{DBI}}+S_{\mathrm{D} 8}^{\mathrm{WZ}} \\
& =\frac{N_{f} T_{8} V_{4}}{g_{s}} \int d^{4} x d U U^{4}\left[\sqrt{f \tau^{\prime 2}+\left(\frac{R_{\mathrm{D} 4}}{U}\right)^{3}\left(f^{-1}-\left(2 \pi l_{s}^{2} A_{0}^{\prime}\right)^{2}\right)}+\kappa B U^{-4} A_{0}^{\prime}\right],
\end{aligned}
$$

where $\kappa=\frac{N_{c}}{12 \pi^{3} l_{s}^{2}} \frac{g_{s} A_{1}}{N_{f} T_{8} V_{4}}$ and $B=B_{23}$. Thus, the influence of the space noncommutativity is brought from WZ-term. The equation of motions for $\tau$ and $A_{0}$ are

$$
\begin{aligned}
& \frac{d}{d U}\left[\frac{U^{4} f \tau^{\prime}}{\sqrt{f \tau^{\prime 2}+\left(\frac{R_{\mathrm{D} 4}}{U}\right)^{3}\left(f^{-1}-\left(2 \pi l_{s}^{2} A_{0}^{\prime}\right)^{2}\right)}}\right]=0, \\
& \frac{d}{d U}\left[\frac{U^{4}\left(\frac{R_{\mathrm{D} 4}}{U}\right)^{3}\left(2 \pi l_{s}^{2} A_{0}^{\prime}\right)}{\sqrt{f \tau^{\prime 2}+\left(\frac{R_{\mathrm{D} 4}}{U}\right)^{3}\left(f^{-1}-\left(2 \pi l_{s}^{2} A_{0}^{\prime}\right)^{2}\right)}}-\frac{\kappa B}{2 \pi l_{s}^{2}}\right]=0,
\end{aligned}
$$

respectively. Integrating the equations of motion (3.5) and (3.6), we obtain

$$
\begin{aligned}
& \left(\tau^{\prime}\right)^{2}=\frac{\left(\frac{R_{\mathrm{D} 4}}{U}\right)^{6}\left\{U_{0}^{8}+\left(C+\frac{\kappa B_{0}}{2 \pi l_{s}^{2}}\right)^{2}\left(\frac{U_{0}}{R_{\mathrm{D} 4}}\right)^{3}\right\} f_{0}}{f^{2}\left[\left(\frac{R_{\mathrm{D} 4}}{U}\right)^{3}\left(U^{8} f-U_{0}^{8} f_{0}\right)+\left(C+\frac{\kappa B}{2 \pi l_{s}^{2}}\right)^{2} f-\left(C+\frac{\kappa B_{0}}{2 \pi l_{s}^{2}}\right)^{2}\left(\frac{U_{0}}{U}\right)^{3} f_{0}\right]}, \\
& \left(2 \pi l_{s}^{2} A_{0}^{\prime}\right)^{2}=\frac{\left(C+\frac{\kappa B}{2 \pi l_{s}^{2}}\right)^{2}}{\left(\frac{R_{\mathrm{D} 4}}{U}\right)^{3}\left(U^{8} f-U_{0}^{8} f_{0}\right)+\left(C+\frac{\kappa B}{2 \pi l_{s}^{2}}\right)^{2} f-\left(C+\frac{\kappa B_{0}}{2 \pi l_{s}^{2}}\right)^{2}\left(\frac{U_{0}}{U}\right)^{3} f_{0}},
\end{aligned}
$$

where $f_{0}=f\left(U_{0}\right), B_{0}=B\left(U_{0}\right)$, and $C$ denotes a constant of integration. In deriving the equations (3.7) and (3.8), we impose the condition $\tau^{\prime}\left(U_{0}\right) \rightarrow \infty$ that corresponds to the connected configuration of the D8-D8-branes. In order to avoid the singularity of $A_{0}^{\prime}$ at $U=U_{0}$, the constant of integration in Eq. (3.8) should be chosen to $C=-\frac{\kappa B_{0}}{2 \pi l_{s}^{2}}$. Since the asymptotic behavior of $A_{0}^{\prime}$ as $U \rightarrow \infty$ is $A_{0}^{\prime} \sim 0$, we obtain

$$
\mu=\lim _{U \rightarrow \infty} A_{0}(U),
$$

where $\mu$ denotes a finite constant. The boundary value $\mu$ coupled to the baryon number density and can be regarded as the baryon number chemical potential [23, 24, 25]. 
Substituting (3.7) and (3.8) into the D8-branes action (3.4), we have

$$
S_{\mathrm{D} 8}^{\mathrm{U}}=\widetilde{T}_{8} \int_{0}^{\infty} d u\left\{u^{5}+b(u)(b(u)-b(1))\right\} \sqrt{\frac{u^{3}}{u^{3} f(u)\left\{u^{5}+(b(u)-b(1))^{2}\right\}-f(1)}},
$$

where $u=\frac{U}{U_{0}}$ is a dimensionless variable, $f(u)=1-\left(\frac{U_{\mathrm{KK}}}{U_{0}}\right)^{3} u^{-3}$, and $\widetilde{T}_{8}=\frac{N_{f} T_{8} V_{4}}{g_{s}}\left(R_{\mathrm{D} 4}^{3} U_{0}^{7}\right)^{1 / 2} \int d^{4} x$. The notation "U" at the upper right of $S_{\mathrm{D} 8}$ denotes the connected configuration of the D8- and $\overline{\mathrm{D}} 8$-branes. The notation $b$ denotes the dimensionless $B$-field defined by

$$
b(u)=\frac{1}{R_{\mathrm{D} 4}^{3 / 2} U_{0}^{5 / 2}} \frac{\kappa B}{2 \pi l_{s}^{2}}=12 \pi^{2} \frac{\left(2 \pi l_{s}^{2} A_{1}\right)}{N_{f} U_{0}} \frac{q^{3 / 2} u^{3}}{1+q^{3} u^{3}},
$$

where $q=U_{0} \theta$ is the dimensionless noncommutativity parameter. When $B$-field is absent, $b$ equals to zero and the effective action (3.10) coincides with the result in ref. [24].

\subsection{High temperature}

The induced metric on the probe D8-branes associated with the deconfining phase takes the form

$$
\begin{aligned}
d s^{2} & =\left(\frac{U}{R_{\mathrm{D} 4}}\right)^{3 / 2}\left(\tilde{f}(U)\left(d t_{E}\right)^{2}+\left(d x^{1}\right)^{2}+h\left\{\left(d x^{2}\right)^{2}+\left(d x^{3}\right)^{2}\right\}\right)+\left(\frac{R_{\mathrm{D} 4}}{U}\right)^{3 / 2} U^{2} d \Omega_{4}^{2} \\
& +\left[\left(\frac{U}{R_{\mathrm{D} 4}}\right)^{3 / 2}\left(\tau^{\prime}(U)\right)^{2}+\left(\frac{R_{\mathrm{D} 4}}{U}\right)^{3 / 2} \frac{1}{\widetilde{f}(U)}\right] d U^{2} .
\end{aligned}
$$

Substituting the determinant of the induced metric (3.12) and the dilaton (2.2) into the effective action of the D8 branes (2.8), we have

$$
\begin{aligned}
S_{\mathrm{D} 8} & =S_{\mathrm{D} 8}^{\mathrm{DBI}}+S_{\mathrm{D} 8}^{\mathrm{WZ}} \\
& =\frac{N_{f} T_{8} V_{4}}{g_{s}} \int d^{4} x d U U^{4}\left[\sqrt{\tilde{f} \tau^{\prime 2}+\left(\frac{R_{\mathrm{D} 4}}{U}\right)^{3}\left(1-\left(2 \pi l_{s}^{2} A_{0}^{\prime}\right)^{2}\right)}+\kappa B U^{-4} A_{0}^{\prime}\right] .
\end{aligned}
$$

The dependence of the effective action of the D8 branes on the $B$-field arises from the WZ term in the same way as in the low-temperature phase. The equations of motion for $\tau$ and $A_{0}$ are

$$
\begin{aligned}
& \frac{d}{d U}\left[\frac{U^{4} \tilde{f} \tau^{\prime}}{\sqrt{\tilde{f} \tau^{\prime 2}+\left(\frac{R_{\mathrm{D} 4}}{U}\right)^{3}\left(1-\left(2 \pi l_{s}^{2} A_{0}^{\prime}\right)^{2}\right)}}\right]=0, \\
& \frac{d}{d U}\left[\frac{U^{4}\left(\frac{R_{\mathrm{D} 4}}{U}\right)^{3}\left(2 \pi l_{s}^{2} A_{0}^{\prime}\right)}{\sqrt{\tilde{f} \tau^{\prime 2}+\left(\frac{R_{\mathrm{D} 4}}{U}\right)^{3}\left(1-\left(2 \pi l_{s}^{2} A_{0}^{\prime}\right)^{2}\right)}}-\frac{\kappa B}{2 \pi l_{s}^{2}}\right]=0,
\end{aligned}
$$


respectively. There is possibility of taking two kinds of the configurations of the D8- and $\overline{\mathrm{D} 8}$ branes in the high temperature. We consider first the connected configuration of the D8- $\overline{\mathrm{D} 8}$ branes. Integrating the equation of motions (3.14) and (3.15) and imposing the condition $\tau^{\prime}(U) \rightarrow \infty$, we have

$$
\begin{gathered}
\left(\tau^{\prime}\right)^{2}=\frac{U_{0}^{8}\left(\frac{R_{\mathrm{D} 4}}{U}\right)^{6} \widetilde{f}_{0}}{\widetilde{f}^{2}\left[\left(\frac{R_{\mathrm{D} 4}}{U}\right)^{3}\left(U^{8} \widetilde{f}-U_{0}^{8} \widetilde{f}_{0}\right)+\left(\frac{\kappa}{2 \pi l_{s}^{2}}\right)^{2}\left(B-B_{0}\right)^{2} \widetilde{f}\right]} \\
\left(2 \pi \alpha^{\prime} A_{0}^{\prime}\right)^{2}=\frac{\widetilde{f}\left(\frac{\kappa}{2 \pi \alpha^{\prime}}\right)^{2}\left(B-B_{0}\right)^{2}}{\left(\frac{R_{\mathrm{D} 4}}{U}\right)^{3}\left(U^{8} \widetilde{f}-U_{0}^{8} \widetilde{f}_{0}\right)+\left(\frac{\kappa}{2 \pi l_{s}^{2}}\right)^{2}\left(B-B_{0}\right)^{2} \widetilde{f}},
\end{gathered}
$$

where $\tilde{f}_{0}=\widetilde{f}\left(U_{0}\right)$. The constant of integration is chosen to avoid the singularity of $A_{0}^{\prime}$ at $U=U_{0}$. Notice that $A_{0}^{\prime}(U)$ becomes always zero and $A_{0}(U)$ takes a constant value when we turn off the $B$-field. By virtue of the presence of non-zero $B$-field, $A_{0}(U)$ is not a constant.

Substitution of (3.16) and (3.17) into the effective action (3.13) leads to

$$
S_{\mathrm{D} 8}^{\mathrm{U}}=\widetilde{T}_{8} \int_{1}^{\infty} d u\left\{u^{5}+b(u)(b(u)-b(1))\right\} \sqrt{\frac{u^{3} \widetilde{f}(u)}{u^{3} \widetilde{f}(u)\left\{u^{5}+(b(u)-b(1))^{2}\right\}-\widetilde{f}(1)}} .
$$

where $u$ and $\widetilde{T}_{8}$ are the same notations as in Eq. (3.10).

The disconnected configuration of the D8- and $\overline{\mathrm{D} 8}$-branes corresponds to a condition $\tau^{\prime}(U)=$ 0 . For the other condition $\tau^{\prime}(U)=0$, we obtain

$$
\left(2 \pi \alpha^{\prime} A_{0}^{\prime}\right)^{2}=\frac{\left(\widetilde{C}+\frac{\kappa B}{2 \pi l_{s}^{2}}\right)^{2}}{U^{8}\left(\frac{R_{\mathrm{D} 4}}{U}\right)^{3}+\left(\widetilde{C}+\frac{\kappa B}{2 \pi l_{s}^{2}}\right)^{2}},
$$

where $\widetilde{C}$ is a constant of integration. For both Eq (3.17) and (3.19), the asymptotic value of $A_{0}^{\prime}$ for $U \rightarrow \infty$ is zero,

$$
\lim _{U \rightarrow \infty}\left(2 \pi \alpha^{\prime} A_{0}^{\prime}\right)^{2}=0 .
$$

From (3.20) the asymptotic value of $A_{0}$ is a constant. We are able to choose the same asymptotic value of $A_{0}$ in both the connected and disconnected configuration as

$$
\lim _{U \rightarrow \infty} A_{0}(U)=\mu .
$$


The constant value $\mu$ is regarded as the chemical potential. From Eq. (3.19) and Eq. (3.21), the chemical potential is expressed in terms of integration with respect to $u$

$$
\mu=A_{0}(U \rightarrow \infty)=\frac{U_{0}}{2 \pi l_{s}^{2}} \int_{u_{T}}^{u} d u^{\prime} \sqrt{\frac{(\widetilde{c}+b)^{2}}{u^{\prime 5}+(\widetilde{c}+b)^{2}}} .
$$

Substituting $\tau^{\prime}(U)=0$ and (3.19) into the action (3.13), we have

$$
S_{\mathrm{D} 8}^{\|}=\widetilde{T}_{8} \int_{u_{T}}^{\infty} d u \frac{u^{5}+b(b+c)}{\sqrt{u^{5}+(b+c)^{2}}},
$$

where $c=\frac{\widetilde{C}}{R_{\mathrm{D} 4}^{3 / 2} U_{0}^{5 / 2}}$ is a dimensionless constant. The notation "\|" at the upper right of $S_{\mathrm{D} 8}$ denotes the disconnected configuration of the D8- and $\overline{\mathrm{D} 8}$-branes. The difference between the two effective actions (3.18) and (3.23) is given by
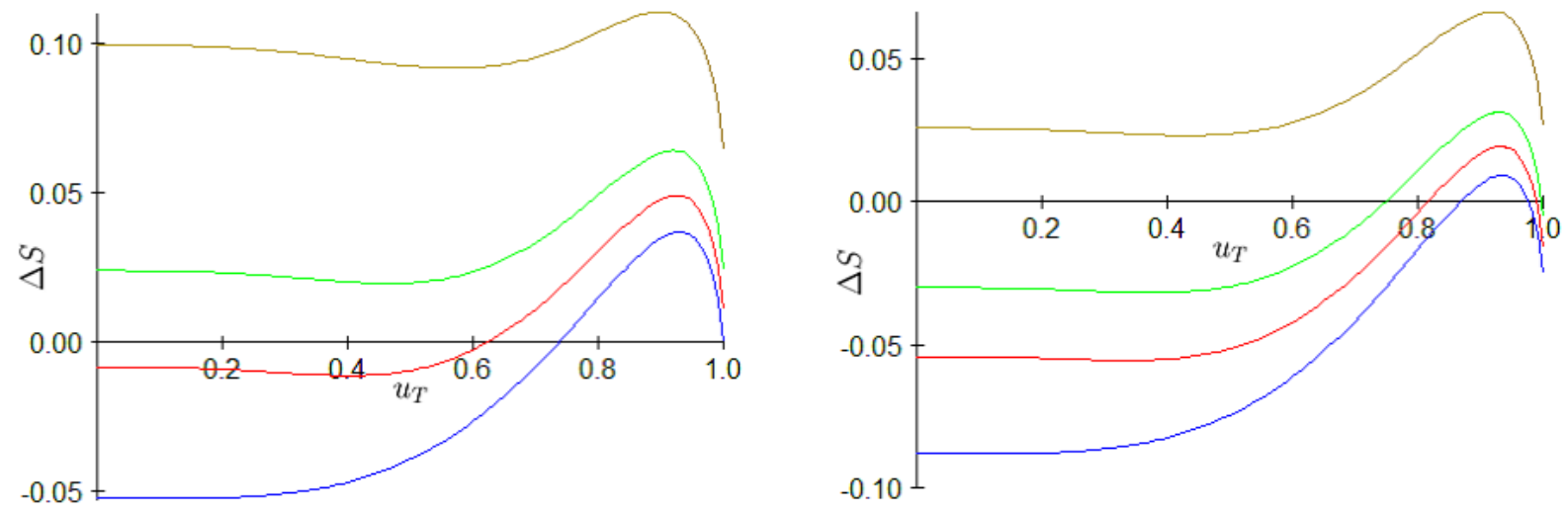

$$
q=0.0
$$

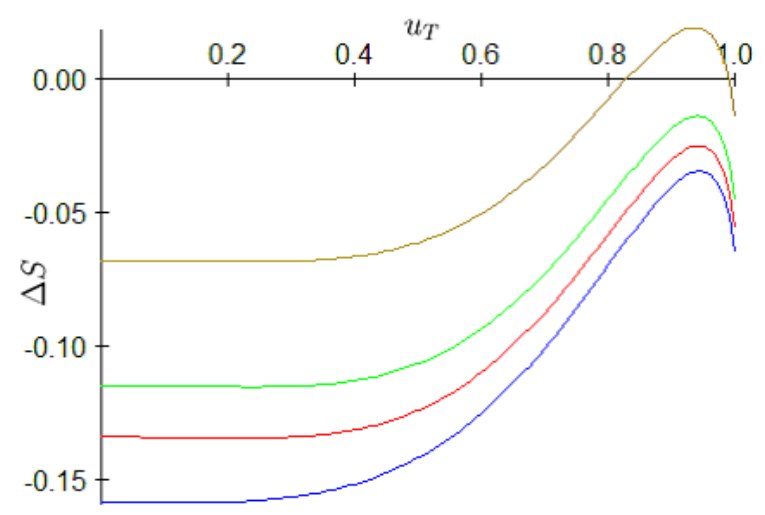

$$
q=1.0
$$

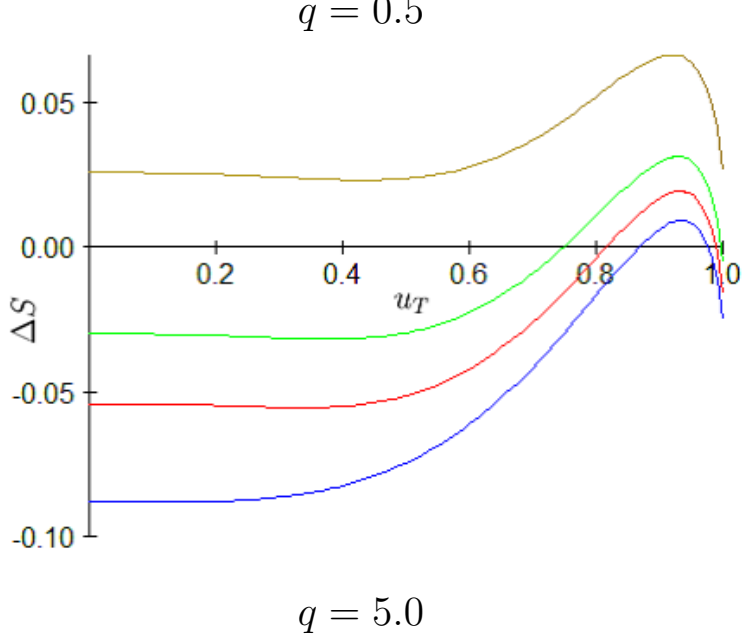

Fig. 3 The numerical calculation of $\Delta S$ as a function of $u_{T}$ for some values of $c$ and $q$. From the bottom to the top each line represents the value of $c$ for $0.0,0.2,0.3,0.5$, respectively. 


$$
\begin{aligned}
\Delta S & \equiv \frac{S_{\mathrm{D} 8}^{\mathrm{U}}-S_{\mathrm{D} 8}^{\|}}{\widetilde{T}_{8}} \\
& =\int_{1}^{\infty} d u\left\{u^{5}+b\left(b-b_{0}\right)\right\} \sqrt{\frac{u^{3} \widetilde{f}(u)}{u^{3} \widetilde{f}(u)\left\{u^{5}+\left(b-b_{0}\right)^{2}\right\}-\widetilde{f}(1)}}-\int_{u_{T}}^{\infty} d u \frac{u^{5}+b(b+c)}{\sqrt{u^{5}+(b+c)^{2}}} .
\end{aligned}
$$

The sign of $\Delta S$ indicates which phase is realized, the chiral symmetry phase or the chiral symmetry broken phase. For $\Delta S<0$, the chiral symmetry broken phase is dominant, while for $\Delta S>0$, the chiral symmetry phase is dominant. The result of the numerical calculation of Eq. (3.24) is shown in Fig. 3.

For $q=0$, the difference $\Delta S$ is reduced to the commutative theory and the behavior of $\Delta S$ is in agreement with the result in Ref. [24]. As the parameter $c$ increases, the phase transition point $u_{T}$ where $\Delta S=0$ decreases for a fixed $q$. In commutative theory, there is a value of $c$ where $\Delta S$ is positive for all values of $u_{T}$ and the chiral symmetry is always restored. In contrast, the phase transition point $u_{T}$ increases with increasing $q$ for a fixed parameter $c$. The noncommutativity parameter $q$ plays the similar role of the constant external $B$-field [32]. In noncommutative theory, there is a value of $c$ where $\Delta S$ is negative for all values of $u_{T}$ and the chiral symmetry is always broken. However, the Fig. 3 shows that the difference $\Delta S$ for $q \rightarrow \infty$ reduces to the one in the commutative theory. This fact is known as a characteristic of the noncommutative theory [14].

Fig. 3 indicates that the chiral symmetry phase structure of this model depends on the parameter $u_{T}, c$ and the dimensionless noncommutativity parameter $q$. This fact allowed us to draw a phase diagram for the noncommutative QCD in the $\left(c, u_{T}\right)$ plane. The phase diagrams with $q=0.0$ and $q=1.0$ are shown in Fig. 4 .
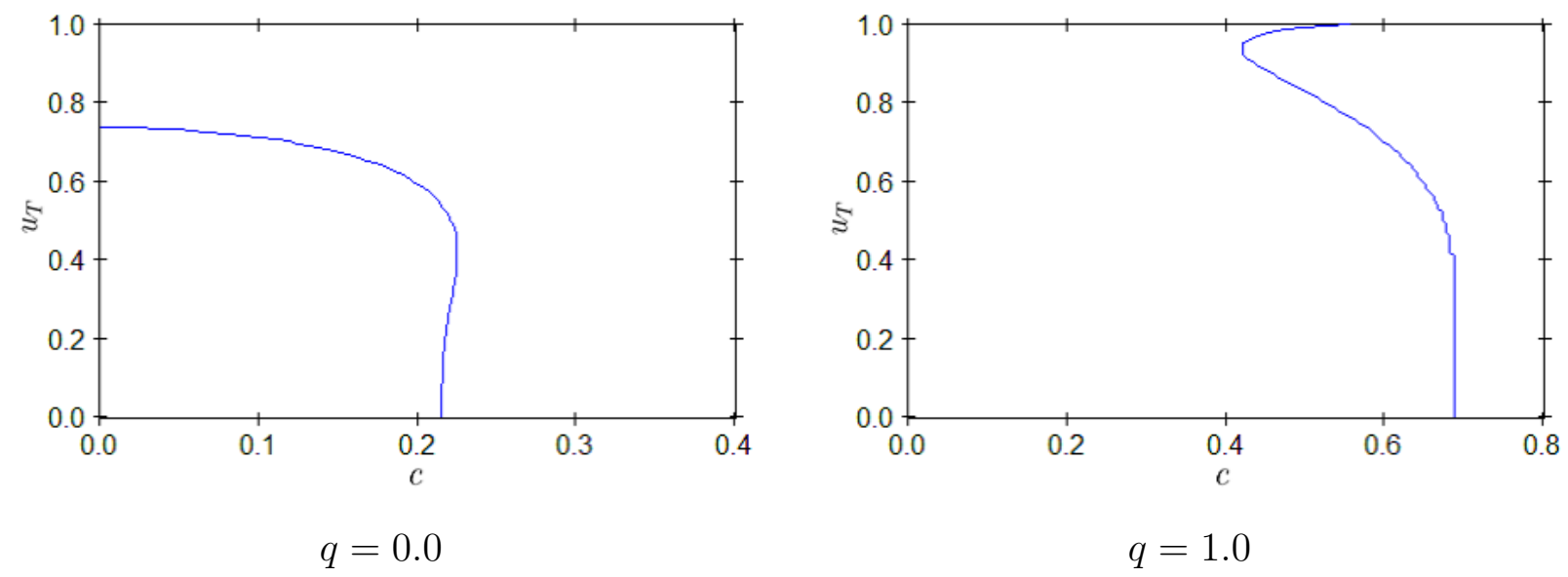

Fig. 4 The phase diagram in the $\left(c, u_{T}\right)$ plane. The area on the left and right side of the curve represents the phase with broken and restored chiral symmetry, respectively. 
In order to translate the phase diagrams shown in Fig. 4 in terms of the temperature $T$ and the chemical potential $\mu$, we rewrite $T$ and $\mu$ as a function with respect to the parameters $u_{T}$ and $c$. From Eq. (3.16), the asymptotic separation $L$ between the D8- and $\overline{\mathrm{D} 8}$-branes is obtained as a functions of $u_{T}$ and $q$ :

$$
L=2 \int_{U_{0}}^{\infty} d U \tau^{\prime}(U)=\left(\frac{R_{\mathrm{D} 4}^{3}}{U_{0}}\right)^{1 / 2} F\left(u_{T}, q\right)
$$

where

$$
F\left(u_{T}, q\right)=\int_{1}^{\infty} d u \frac{2}{\sqrt{u^{3} \widetilde{f}(u)}} \sqrt{\frac{\left\{1+\left(b-b_{0}\right)^{2}\right\} \widetilde{f}(1)}{u^{8} \widetilde{f}(u)-\widetilde{f}(1)+\left(b-b_{0}\right)^{2} u^{3} \widetilde{f}(u)}}
$$

The temperature $T$ given in Eq. (2.5) is expressed in terms of $L$ by eliminating $U_{0}$ :

$$
T=\frac{3}{4 \pi}\left(\frac{u_{T} U_{0}}{R_{\mathrm{D} 4}^{3}}\right)^{1 / 2}=\frac{3}{4 \pi} \frac{\sqrt{u_{T}}}{L} F\left(u_{T}, q\right) .
$$

Notice that the temperature $T$ depends on not only the parameter $u_{T}$ but also the noncommutativity parameter $q$. Remember the confinement/deconfinement phase transition happens when $T=1 / 2 \pi R$. If the ratio $L / R$ is smaller than $\frac{3}{2} \sqrt{u_{T}} F\left(u_{T}, q\right)$, there exist the intermediatetemperature phase. The chemical potential given in Eq. (3.22) is also expressed in terms of $L$ as

$$
\mu=\frac{R_{\mathrm{D} 4}^{3}}{2 \pi l_{s}^{2} L^{2}} F\left(u_{T}, q\right)^{2} \int_{u_{T}}^{u} d u^{\prime} \sqrt{\frac{(\widetilde{c}+b)^{2}}{u^{\prime 5}+(\widetilde{c}+b)^{2}}} .
$$

Eq. (3.28) shows that the chemical potential depends on not only the parameters $c$ and $u_{T}$ but also the noncommutativity parameter $q$. The dimensionless temperature $\widetilde{T}$ and the dimensionless chemical potential $\widetilde{\mu}$ can be defined by using the asymptotic separation $L$ as

$$
\begin{aligned}
& \widetilde{T}=L T=\frac{3}{4 \pi} \sqrt{u_{T}} F\left(u_{T}, q\right), \\
& \widetilde{\mu}=\frac{2 \pi l_{s}^{2} L^{2}}{R_{\mathrm{D} 4}^{3}} \mu=F\left(u_{T}, q\right)^{2} \int_{u_{T}}^{u} d u^{\prime} \sqrt{\frac{(\widetilde{c}+b)^{2}}{u^{\prime 5}+(\widetilde{c}+b)^{2}}} .
\end{aligned}
$$

The dimensionless temperature $\widetilde{T}$ and the dimensionless chemical potential $\widetilde{\mu}$, as function with respect to the noncommutativity parameter $q$, are shown in Fig. 5 and Fig. 6, respectively. 


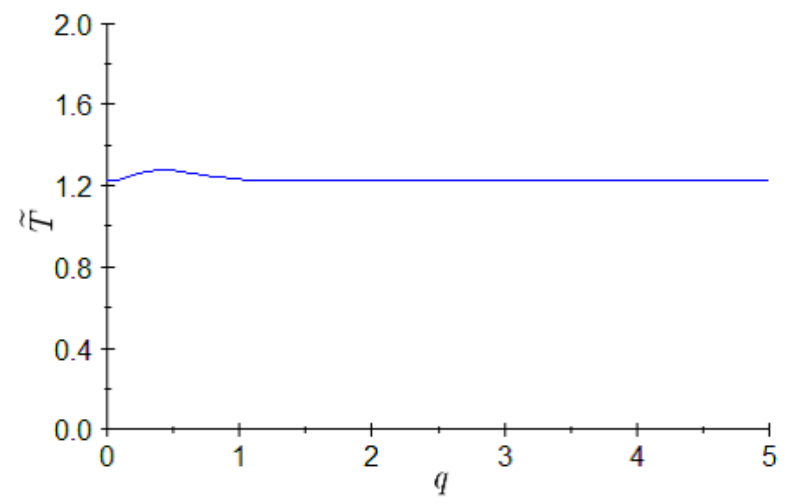

Fig. 5 The dimensionless temperature $\widetilde{T}$ as a function of $q$ for $u_{T}=0.5$. The temperature is hardly affected by the noncommutativity parameter.
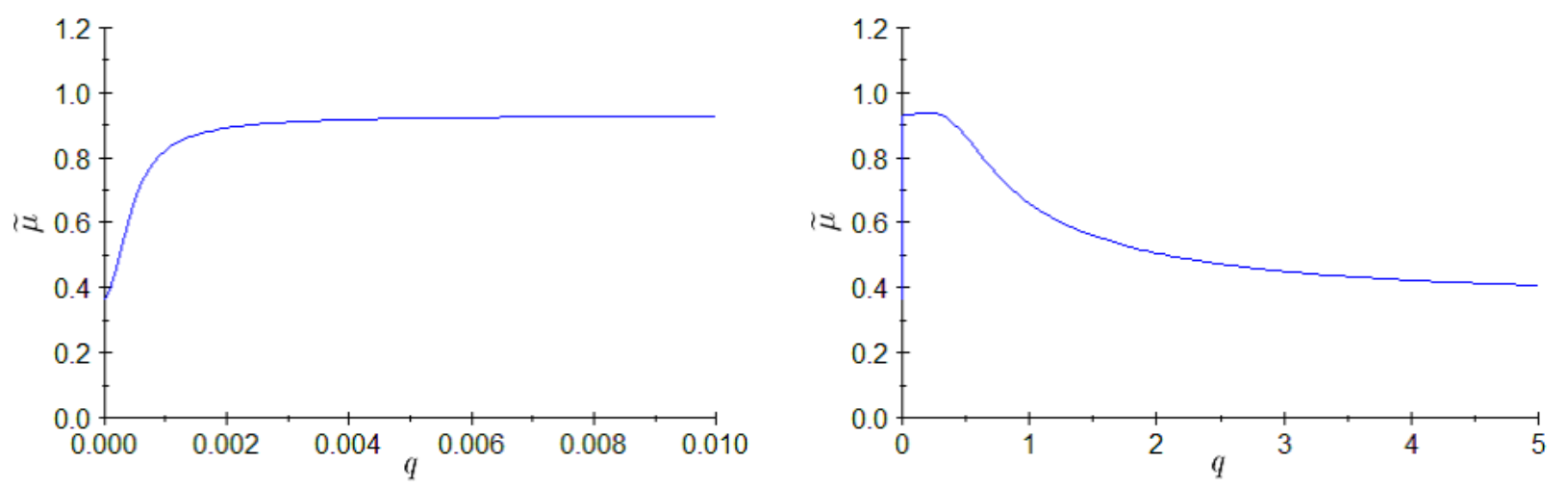

Fig. 6 The dimensionless chemical potential $\widetilde{\mu}$ as a function of $q$ for $u_{T}=0.5$ and $c=0.5$. As $q$ increases, $\widetilde{\mu}$ approaches the one in the commutative theory.

Although the asymptotic separation $L$ can be regarded as approximately constant with fixed noncommutativity parameter, $L$ rapidly shrinks as $u_{T} \rightarrow 1$. The behavior of the dimensionless asymptotic separation $\widetilde{L}=\left(\frac{U_{0}}{R_{\mathrm{D} 4}^{3}}\right)^{1 / 2} L$ as a function of $u_{T}$ is shown in Fig. 7 . 


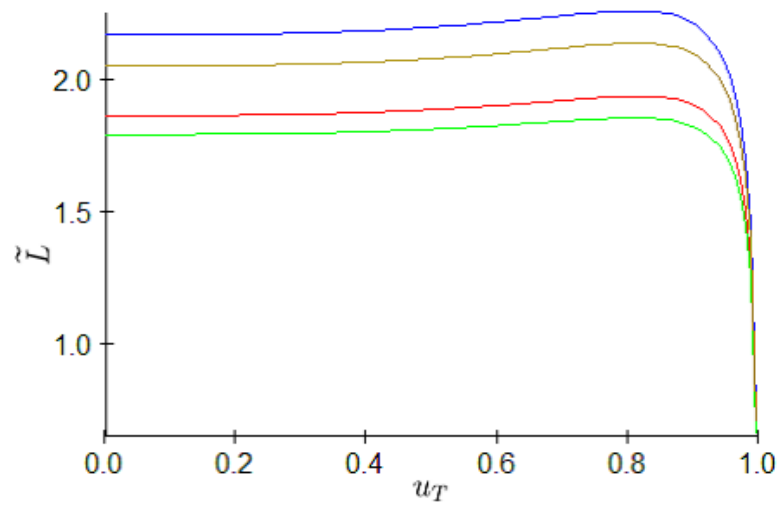

Fig. 7 The dimensionless asymptotic separation $\widetilde{L}=\left(\frac{U_{0}}{R_{\mathrm{D} 4}^{3}}\right)^{1 / 2} L$ as a function of $u_{T}$ for some value of $q$. From the top to the bottom each line represents the value of $q$ for 0.0, 5.0, 0.5, 1.0, respectively. $\widetilde{L}$ for $q \rightarrow \infty$ reduces to the one in the commutative theory.

By utilizing Eqs. (3.27) and (3.28), we can draw the phase diagram in terms of the temperature $T$ and the chemical potential $\mu$. The phase diagram in the $(\widetilde{\mu}, \widetilde{T})$ plane is shown in Fig. 8.
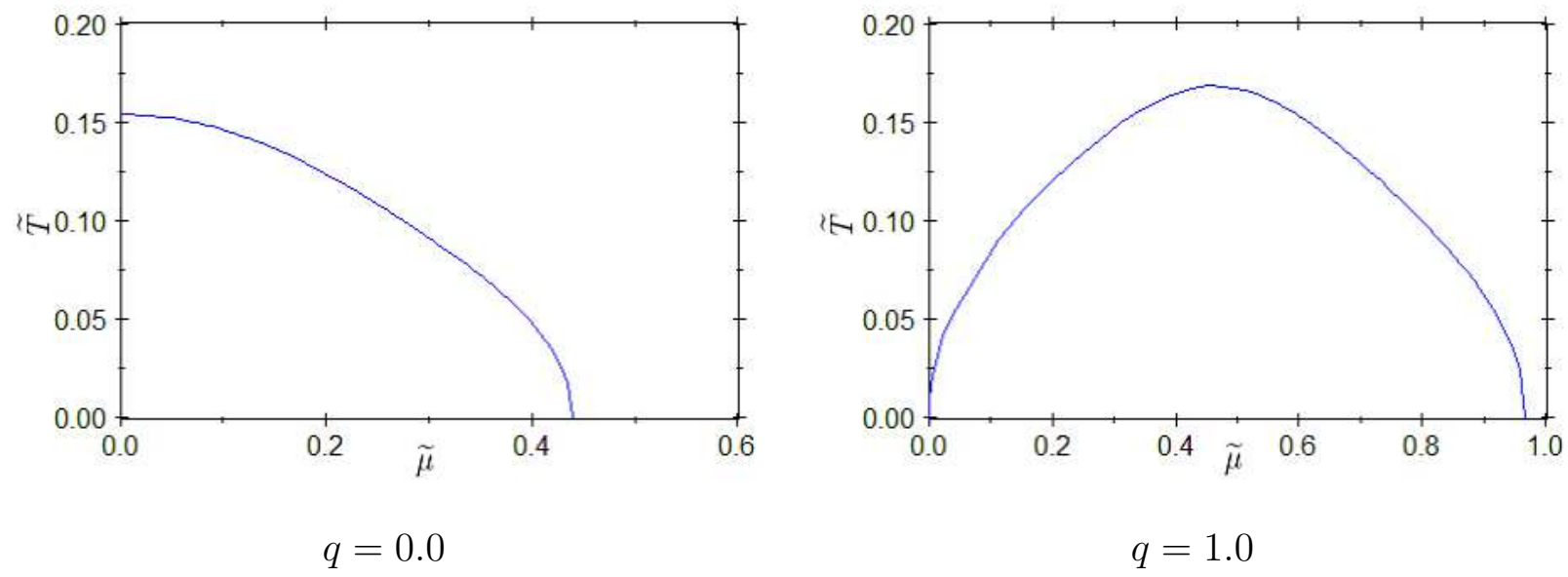

Fig. 8 The phase diagram in the $(\widetilde{\mu}, \widetilde{T})$ plane. The area containing the origin represents the phase with broken chiral symmetry, and the other area represents the phase with restored chiral symmetry.

Fig. 8 indicates that the phase diagram is modified by the space noncommutativity. Although the chiral phase transition occurs at high-temperature and high-chemical potential in 
the noncommutative theory, the space noncommutativity plays a role of increasing the critical value of the chemical potential. The modification of the phase diagram around the origin in the noncommutative theory is brought about the shrinking of the asymptotic separation $L$.

\section{Conclusions and Discussions}

In this paper, we have constructed a noncommutative deformation of the holographic QCD (Sakai-Sugimoto) model after the prescription of Arean-Paredes-Ramallo [14]. In the same way as D3-D7 brane system, WZ terms in the effective action of probe D8-brane play the important role of bringing the space noncommutativity in the gauge theory side. Both the temperature $T$ and the chemical potential $\mu$ in the gauge theory side depend on the noncommutativity parameter due to the noncommutative deformation. Fig. 5 and 6 show that although the dependence on the noncommutativity parameter of the temperature is slight, that of the chemical potential is not very small. The critical value of the chemical potential in the noncommutative space tends to be larger than that in the commutative space.

The space noncommutativity has an effect on an aspect of the chiral phase transition for the holographic QCD at finite temperature. In general, intermediate temperature phase, in which the gluons deconfine but the chiral symmetry remains broken, is easy to be realized in some region of the noncommutativity parameter. The phase diagram of the noncommutative theory shows the phase transition occurs in "high"-chemical potential region in comparison with the commutative theory. The higher critical values of the chemical potential are caused by the effect that space noncommutativity plays a role of increasing the critical value of the chemical potential. The fact indicates that the chiral symmetry restoration tends to be hard to happen in the noncommutative theory.

On the other hand, the phase diagram of the noncommutative theory shows the phase transition also occurs in low-temperature and low chemical potential region. It can be interpreted that the phase transition in low-temperature and low chemical potential region is caused by the rapidly shrinking of the asymptotic separation $L$, rather than the space noncommutativity. More exact evaluation will be required for the phase diagram of the noncommutative theory in low-temperature and low chemical potential region.

The magnitude of noncommutativity parameter $\theta$ denotes the degree of space noncommutativity in the noncommutative theory. The noncommutative QCD theory reduces to the commutative one when the noncommutativity parameter tends to zero. However, the noncommutative QCD theory also reduces to the commutative one in the large noncommutativity parameter limit. This property reminds us Morita equivalence between noncommutative tori [34].

The noncommutative QCD tends to realize easily the intermediate temperature phase than 
ordinary QCD. In the intermediate temperature phase, there still be bound state of the hadron degrees of freedom. The property of the hadron in QCD at finite temperature can be analyzed by the holographic method. In the intermediate temperature phase, the masses of the low spin meson are temperature dependent and there is a dissociation phenomenon of the large spin mesons [22]. It is interesting to analyze the property of the hadron in the noncommutative QCD at finite temperature and to make clear what kind of influence has the noncommutativity parameter had on the property of the hadron.

The UV/IR mixing is well known as distinctive features of noncommutative field theories. The UV/IR mixing appears to be the qualitative difference between ordinary and noncommutative field theory. The difference in chiral phase structure between ordinary and noncommutative QCD might be related to the UV/IR mixing. We hope to discuss this subject in the future.

\section{Acknowledgments}

We are grateful to A. Sugamoto for useful discussions and comments. One of us (T.N.) would like to thank members of the Physics Depertment at College of Engineering, Nihon University for their encouragements. 


\section{References}

[1] A. Connes, M. R. Douglas, A. Schwarz, Noncommutative Geometry and Matrix Theory: Compactification on Tori, JHEP 02 (1998) 003, arXiv:hep-th/9711162.

[2] M. R. Douglas and C. Hull, D-branes and the Noncommutative Torus, JHEP 02 (1998) 008, arXiv:hep-th/9711165.

[3] F. Ardalan, H. Arfaei and M.M. Sheikh-Jabbari, Noncommutative Geometry From Strings and Branes, JHEP 02 (1999) 016, arXiv:hep-th/9810072.

[4] M.M. Sheikh-Jabbari, Super Yang-Mills Theory on Noncommutative Torus from Open Strings Interactions, Phys. Lett. B450 (1999) 119-125, arXiv:hep-th/9810179.

[5] N. Seiberg and E. Witten, String Theory and Noncommutative Geometry, JHEP 9909 (1999) 032, arXiv:hep-th/9908142.

[6] S. Minwalla, M. V. Raamsdonk and N. Seiberg, Noncommutative perturbative dynamics JHEP 0002 (2000) 020, arXiv:hep-th/9912072

[7] A. Hashimoto and N. Itzhaki, Non-Commutative Yang-Mills and the AdS/CFT Correspondence, Phys. Lett. B465 (1999) 142-147, arXiv:hep-th/9907166.

[8] J. M. Maldacena and J. G. Russo, Large N Limit of Non-Commutative Gauge Theories, JHEP 9909 (1999) 025, arXiv:hep-th/9908134.

[9] M. Alishahiha, Y. Oz and M.M. Sheikh-Jabbari, Supergravity and Large N Noncommutative Field Theories, JHEP 9911 (1999) 007, arXiv:hep-th/9909215.

[10] A. Dhar and Y. Kitazawa, "Wilson loops in strongly coupled noncommutative gauge theories," Phys. Rev. D63 (2001) 125005, arXiv:hep-th/0010256.

[11] S. Lee and S. Sin, "Wilson Loop and Dimensional Reduction in Non-Commutative Gauge Theories," Phys. Rev. D64 (2001) 086002, arXiv:hep-th/0104232.

[12] H. Takahashi, T. Nakajima and K. Suzuki, "D1/D5 system and Wilson Loops in (Non-)commutative Gauge Theories," Phys. Lett. B546 (2002) 273, arXiv:hep-th/0206081.

[13] T. Nakajima, K. Suzuki and H. Takahashi, "Glueball mass spectra for supergravity duals of noncommutative gauge theories," JHEP 0601 (2006) 016, arXiv:hep-th/0508054. 
[14] D. Arean, A. Paredes and A.V. Ramallo, Adding flavor to the gravity dual of noncommutative gauge theories, JHEP 0508 (2005) 017, arXiv:hep-th/0505181.

[15] J. Erdmenger, N. Evans, I. Kirsch and E. Threlfall, Mesons in Gauge/Gravity Duals - A Review, Eur. Phys. J. A35 (2008) 81-133, arXiv:0711.4467 [hep-th].

[16] D. Mateos, String Theory and Quantum Chromodynamics, Class. Quant. Grav. 24 (2007) S713-S740, arXiv:0709.1523 [hep-th].

[17] K. Peeters and M. Zamaklar, The string/gauge theory correspondence in QCD, Eur. Phys. J. ST152 (2007) 113-138, arXiv:0708.1502 [hep-ph].

[18] T. Sakai and S. Sugimoto, Low energy hadron physics in holographic QCD, Prog. Theor. Phys. 113 (2005) 843-882, arXiv:hep-th/0412141.

[19] T. Sakai and S. Sugimoto, More on a holographic dual of QCD, Prog. Theor. Phys. 114 (2006) 1083-1118, arXiv:hep-th/0507073.

[20] O. Aharony, J. Sonnenschein and S. Yankielowicz, A holographic model of deconfinement and chiral symmetry restoration, Annals Phys. 322 (2007) 1420-1443, arXiv:hep-th/0604161.

[21] A. Parnachev and D. A. Sahakyan, Chiral Phase Transition from String Theory, Phys.Rev.Lett. 97 (2006) 111601, arXiv:hep-th/0604173,

[22] K. Peeters, J. Sonnenschein and M. Zamaklar, Holographic melting and related properties of mesons in a quark gluon plasma, Phys. Rev. D74 (2006) 106008, arXiv:hep-th/0606195.

[23] K. Y. Kim, S.-j. Sin and I. Zahed, Dense Hadronic Matter in Holographic QCD, arXiv:hep-th/0608046.

[24] N. Horigome and Y. Tanii, Holographic chiral phase transition with chemical potential, JHEP 0701 (2007) 072, arXiv:hep-th/0608198.

[25] A. Parnachev and D. A. Sahakyan, Photoemission with Chemical Potential from QCD Gravity Dual, Nucl. Phys. B768 (2007) 177-192, arXiv:hep-th/0610247.

[26] D. Yamada, Sakai-Sugimoto Model at High Density, JHEP 0810 (2008) 020, arXiv:0707.0101.

[27] Y. Seo, S.-j. Sin and W.-s. Xu, Note on a non-critical holographic model with a magnetic field, Phys. Rev. D81 (2010) 066001, arXiv:0910.2661[hep-th]. 
[28] T. Matsuo, D. Tomino and Wen-Yu Wen, Drag force in SYM plasma with B field from AdS/CFT, JHEP 10 (2006) 055, arXiv:hep-th/0607178

[29] K. L. Panigrahi and S. Roy, Drag force in a hot non-relativistic, non-commutative Yang-Mills plasma, arXiv:1001.2904 [hep-th].

[30] J. Sadeghi and B. Pourhassan, Energy loss and jet quenching parameter in a thermal non-relativistic, non-commutative Yang-Mills plasma, arXiv:1002.1596 [hep-th].

[31] O. Bergman, G. Lifschytz and M. Lippert, Response of Holographic QCD to Electric and Magnetic Fields, JHEP 0805 (2008) 007, arXiv:0802.3720[hep-th].

[32] C. V. Johnson and A. Kundu, External Fields and Chiral Symmetry Breaking in the Sakai-Sugimoto Model, JHEP 0812 (2008) 053, arXiv:0803.0038[hep-th].

[33] W.-H. Huang, Supergravity Solution Dual to Holographic Gauge Theory with Maxwell Magnetic Field, arXiv:0904.2328[hep-th].

[34] M. R. Douglas and N. A. Nekrasov, Noncommutative Field Theory, Rev. Mod. Phys. 73 (2001) 977-1029, arXiv:hep-th/0106048.

[35] R.J. Szabo, Quantum field theory on noncommutative spaces, Phys. Rept. 378 (2003) 207-299, arXiv:hep-th/0109162. 